Tavakolifard, M., Atle Gulla, J., Almeroth, K. C., Hopfgartner, F., Kille, B., Plumbaum, T., Lommatzsch, A., Brodt, T., Bucko, A., Heintz, T.

\title{
Workshop and challenge on news recommender systems
}

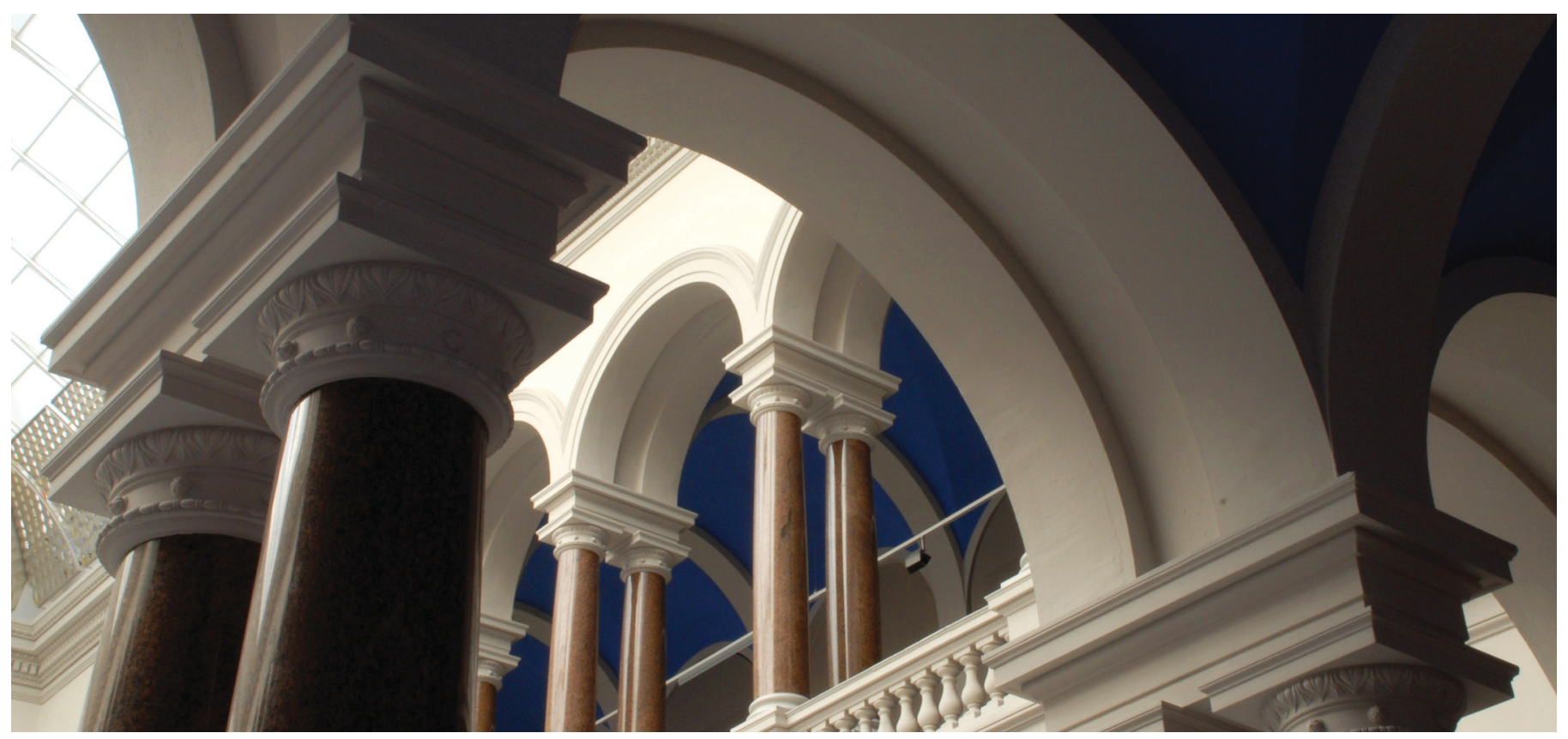

(C) Owner/Author I ACM 2013. This is the author's version of the work. It is posted here for your personal use. Not for redistribution. The definitive Version of Record was published in Proceedings of the 7th ACM conference on Recommender systems, http://dx.doi.org/10.1145/2507157.2508004.

Tavakolifard, M., Heintz, T., Gulla, J. A., Almeroth, K. C., Hopfgartner, F., Kille, B., ... Bucko, A. (2013). Workshop and challenge on news recommender systems. In Proceedings of the 7th ACM conference on Recommender systems - RecSys '13. ACM Press. https://doi.org/10.1145/2507157.2508004 


\section{Workshop and Challenge on News Recommender Systems}

\author{
Mozhgan Tavakolifard ${ }^{1}$, Jon Atle Gulla ${ }^{1}$, \\ Kevin C. Almeroth², Frank Hopfgartner ${ }^{3}$ \\ Workshop organizers \\ ${ }^{1}$ Norwegian University of Science \& Techn. \\ \{mozhgan, jag\}@idi.ntnu.no \\ 2University of California, Santa Barbara, \\ almeroth@cs.ucsb.edu \\ ${ }^{3}$ Technische Universität Berlin, \\ frank.hopfgartner@tu-berlin.de
}

\author{
Benjamin Kille ${ }^{1}$, Till Plumbaum ${ }^{1}$, \\ Andreas Lommatzsch ${ }^{1}$, Torben Brodt ${ }^{2}$, \\ Arthur Bucko ${ }^{2}$, Tobias Heintz ${ }^{2}$ \\ Challenge organizers \\ ${ }^{1}$ Technische Universität Berlin \\ \{firstname.lastname\}@tu-berlin.de \\ 2Plista GmbH, \{tb, ab, thz\}@plista.com
}

\begin{abstract}
Recommending news articles entails additional requirements to recommender systems. Such requirements include special consumption patterns, fluctuating item collections, and highly sparse user profiles. This workshop (NRS'13@RecSys) brought together researchers and practitioners around the topics of designing and evaluating novel news recommender systems. Additionally, we offered a challenge allowing participants to evaluate their recommendation algorithms with actual user feedback.
\end{abstract}

\section{Categories and Subject Descriptors}

H.3.3 [Information Search and Retrieval]

\section{General Terms}

Algorithm, Experimentation, Theory

\section{Keywords}

News; Recommender Systems

\section{OVERVIEW}

News article recommendation differs in several ways from other well-known types of recommender systems such as for music and movies: 1) Freshness represents an important aspect. Sometimes, freshness is deemed more important than relevancy. On one hand, users are usually interested in most recent news. On the other hand, users sometimes are interested to read previous relevant articles [4, 8, 9, 11, 16, 17], 2) Large volume of news articles make this domain different from other types of Web objects. News articles tend to be in flood within a short period of time, requiring much more computation for recommendation [3, 6, 9, 13, 14], 3) Similarity between news articles does not necessarily reflect their relatedness. For instance, two news articles might share a majority of words. Still, their actual topic might differ [12], 4) News are typically published in a rather unstructured format [14].
In contrast, structured data such as social graphs facilitate preprocessing steps, 5) Most news articles describe the occurring of specific events. Online news readers' interests are highly correlated to so-called named entities. Such named entities describe who was involved in the event. News readers might have special entity preferences on some particular events which recommender systems can barely predict. Still, we struggle detecting such preferences due to little information on the user available, 6 ) Serendipities (i. e., variety in recommended news articles) represent a crucial property of a news recommender system. Contrarily to music recommendations, users avoid to re-consume an item. Thus, news recommender systems are required to provide diverse sets of items in order to assure not to recommend monotonously. Users might have great interest in news articles for which the content does not match with the information the system has gathered about the user $[2,5,7,15], 7)$ Breaking or trendy news might have a high relevance even though the appear completely unrelated to the individual user profile [11]. Popularity and recency of news articles change dramatically along with the time, which differentiates other news items, such as products and movies, rendering traditional recommendation methods ineffective, 8) it has not yet established consensus on how to evaluate news recommender systems. We typically observe implicit preferences as users interact with news portals. Those preferences do not exhibit a graded relevance. Thus, well-established evaluation criteria based on ratings (e.g., root mean squared error) cannot be applied, 9) Recommender systems are known to struggle with socalled "cold-start users" (i. e., users form whom no preferences are available yet). News portals typically refrain to require users to login prior to read news articles. Hence, there is a large fraction of users who appear to be "cold-start users" $[4,6,8,9,10]$. 10) The lack of sufficiently many interaction to established trustworthy user profiles entails further challenges. Inferring interest signals suffers from incomplete profiles. News providers do generally not require their users to create own user profiles and users consume news articles anonymously. Therefore, news recommender systems have to cope without explicit user profiles. Tracking users suffers from changing session identifiers, access limiting browser setups, and shared computers. This increases the sparsity of data as well $[3,9,14]$.

Another challenge related to the context arises from the increasingly frequent use of mobile devices to read news articles such as tablets and smart phones [1]. Those have a limited space available to display news and related recommendations. News recommender systems have to deal with this issue thus applying suited layout mechanisms to fit the content to the available screen. 
Finally, news recommender systems face numerous technical challenges. Those challenges include minimizing response time improve user experience, scaling to the large amount of requests to avoid time outs, providing flexibility to incorporate new recommendation methods or adjust parameter settings for existing implementations, and guarantee a reliable service whom user can access at any time.

\section{SCOPE}

This workshop aimed at creating an interdisciplinary community focusing on the design issues for news recommender systems and promoting collaboration opportunities between researchers and practitioners. We brought together researchers as well as practitioners around the topics of designing and evaluating novel news recommender systems in order to: (1) share research on news recommendation techniques and evaluation methodologies, (2) explore key challenges in the area, and (3) identify emerging topics.

\subsection{Workshop}

The topics of papers submitted to the workshop can be summarized as follows: recommendation techniques suited for news recommendation, tracking the evolution of news over time, evaluation approaches, the interplay of news and social-media data, user interface for news portals and their implications to news recommendation, news mining and analytics, news reader behavioral models, semantic and news context analysis, user profiling and preference elicitation, news recommendations on mobile platforms, and information retrieval in news collections. The papers covered a wide range of different perspective on the field. The accepted papers included a practitioners perspective based on an actual news recommender systems and their data on the one hand. On the other hand, a survey on the state-of-the-art of news recommender systems and their inherent challenges was conducted to illustrate the research perspective.

\subsection{Challenge}

Prior to the workshop, we offered researchers as well as practitioners the chance to interact with a real-time news recommender system. We released an off-line data set for bootstrapping purposes. This data set contained more than 84 million interactions recorded over a 4 week period. Subsequently, participants could directly interact with a news recommender system providing news on several news portals in the time frame from August 15 - September 15,2013 . The participants received actual recommendation request and transmit recommendations. Thus, we were able to mimic the actual requirements for news recommender systems. Those requirements included to handle a large amount of request in real-time with a fixed response time limit. At the workshop we presented evaluation results of the challenge.

\section{FURTHER INFORMATION}

The workshop material (list of accepted papers, invited talk, and the workshop schedule) can be found at the workshop webpage: https://sites.google.com/site/newsrec2013/home. The workshop proceedings are published in the ACM International Conference Proceedings Series (ICPS). Papers related could not be submitted before the conference deadline. The challenge-related papers which have been accepted are published as CEUR proceedings.

\section{REFERENCES}

[1] D. Billsus and M. Pazzani. User modeling for adaptive news access. User modeling and user-adapted interaction, 10(2):147-180, 2000

[2] H. L. Borges and A. C. Lorena. A survey on recommender systems for news data. In Smart Information and Knowledge Management, pages 129-151. Springer, 2010.

[3] A. S. Das, M. Datar, A. Garg, and S. Rajaram. Google news personalization: scalable online collaborative filtering. In Proceedings of the 16th international conference on World Wide Web, pages 271-280. ACM, 2007.

[4] B. Fortuna, C. Fortuna, and D. Mladenić. Real-time news recommender system. In Machine Learning and Knowledge Discovery in Databases, pages 583-586. Springer, 2010.

[5] E. Gabrilovich, S. Dumais, and E. Horvitz. Newsjunkie: providing personalized newsfeeds via analysis of information novelty. In Proceedings of the 13th international conference on World Wide Web, pages 482-490. ACM, 2004.

[6] F. Garcin, C. Dimitrakakis, and B. Faltings. Personalized news recommendation with context trees. arXiv preprint arXiv:1303.0665, 2013.

[7] L. Iaquinta, M. De Gemmis, P. Lops, G. Semeraro, M. Filannino, and P. Molino. Introducing serendipity in a content-based recommender system. In Hybrid Intelligent Systems, 2008. HIS'08. Eighth International Conference on, pages 168-173. IEEE, 2008.

[8] H. J. Lee and S. J. Park. Moners: A news recommender for the mobile web. Expert Systems with Applications, 32(1):143-150, 2007.

[9] L. Li, D. Wang, T. Li, D. Knox, and B. Padmanabhan. Scene: a scalable two-stage personalized news recommendation system. In SIGIR, pages 125-134, 2011.

[10] C. Lin, R. Xie, L. Li, Z. Huang, and T. Li. Premise: personalized news recommendation via implicit social experts. In Proceedings of the 21st ACM international conference on Information and knowledge management, pages 1607-1611. ACM, 2012.

[11] J. Liu, P. Dolan, and E. R. Pedersen. Personalized news recommendation based on click behavior. In Proceedings of the 15th international conference on Intelligent user interfaces, pages 31-40. ACM, 2010.

[12] Y. Lv, T. Moon, P. Kolari, Z. Zheng, X. Wang, and Y. Chang. Learning to model relatedness for news recommendation. In Proceedings of the 20th international conference on World wide web, pages 57-66. ACM, 2011.

[13] P. Resnick, N. Iacovou, M. Suchak, P. Bergstrom, and J. Riedl. Grouplens: an open architecture for collaborative filtering of netnews. In Proceedings of the ACM conference on Computer supported cooperative work, pages 175-186. ACM, 1994.

[14] Saranya.k.g and G. S. Sadhasivam. A personalized online news recommendation system. International Journal of Computer Applications, 57(18):6-14, November 2012.

[15] V. Vinod, R. A. Jim, and C. P. S. George. News sync: Three reasons to visualize news better. HCIR 2010, page 45, 2010.

[16] H. Wen, L. Fang, and L. Guan. A hybrid approach for personalized recommendation of news on the web. Expert Systems with Applications, 39(5):5806-5814, 2012.

[17] K. F. Yeung and Y. Yang. A proactive personalized mobile news recommendation system. In Developments in E-systems Engineering (DESE), 2010, pages 207-212. IEEE, 2010 . 